\title{
Moving Target Tracking of Extended Nonholonomic Chained-Form Systems via Finite-Time Switching Control
}

\author{
Hua Chen ${ }^{1},{ }^{1}$ Lei Chen, ${ }^{2}$ and Fei Tong ${ }^{2}$ \\ ${ }^{1}$ Mathematics and Physics Department, Hohai University, Changzhou Campus, Changzhou 213022, China \\ ${ }^{2}$ College of Internet of Things of Engineering, Hohai University, Changzhou 213022, China \\ Correspondence should be addressed to Hua Chen; chenhual12@163.com
}

Received 20 May 2018; Revised 14 July 2018; Accepted 29 July 2018; Published 14 August 2018

Academic Editor: Yuriy Rogovchenko

Copyright (c) 2018 Hua Chen et al. This is an open access article distributed under the Creative Commons Attribution License, which permits unrestricted use, distribution, and reproduction in any medium, provided the original work is properly cited.

\begin{abstract}
A finite-time switching control scheme is presented for tracking a practical moving target of extended nonholonomic chainedform systems. Firstly, a dynamic output tracking error model is proposed combining moving target and extended nonholonomic chained-form systems. Secondly, two decoupled subsystems are considered for the tracking error systems, based on which the rigorous convergence and stability analysis are proposed by applying the finite-time stability control theory and switching design methods. Finally, the effectiveness of the proposed finite-time switching control approach is performed according to the further simulation results.
\end{abstract}

\section{Introduction}

In the past few years, nonholonomic systems especially their extended version $([1,2])$ have received considerable attention. Large amounts of research have been conducted adaptively, such as wheeled mobile robots, free-floating space robots, and tractor-trailer systems depicted in articles $[3,4]$. Plenty of methods with respect to stabilizing a control system have been continuously presented as well. Zhiqiang Miao et al. [5] recently have implemented some research on moving target with multiple nonholonomic robots using backstepping design to ensure asymptotic convergence of the robot group to the desired proposal. Nevertheless, when it comes to the tracking problem, there still exist great challenges for the available tracking error systems to stabilize using smooth feedback control laws among the control community [6-9]. Therefore, the tracking control of extended nonholonomic chained-form systems still remains as the concentrate focuses among the research fields.

To settle the mentioned tracking problem, several finitetime control approaches have been proposed continuously [10-16]. For example, terminal sliding-mode control introducing a fractional power term in the sliding surface has been designed to realize finite-time convergence and high-precision performance with high probability [10, 11]. Some time ago, adaptive robust control technique, which can be used in the repetitive learning finite-time controller design, has been presented [12]. Finite-time control for hyperchaotic Lorenz-Stenflo systems with parameter uncertainties [13], global set stabilization of the spacecraft attitude [14], a class of planar systems [15], and some other different investigations have also been proposed, relevantly. Furthermore, Hong et al. [16, 17] have initiated research robust stabilization, aiming at achieving trajectory tracking of extended chained systems via finite-time control method, and then the recursive terminal sliding-mode was introduced concentrating on external disturbances.

As for tracking control problem, most of trajectory tracking control schemes focus exclusively on guaranteeing asymptotic convergence of the trajectory tracking errors as certified in the existing literatures [18-21]. Practically, it is often required that desired trajectory tracking errors should approach zero in a finite time to guarantee perfect tracking performance. Therefore, a finite-time nonlinear controller satisfying the distance and bearing angle constraints was designed for nonholonomic ground vehicles to track a moving target with desired distance and bearing angles, which has been proofed in detail by Wang et al. [22] 
As for tracking issues with moving targets, Mohammad et al. [23] ever developed a three-dimensional guidance and control algorithm to decrease the probability of missing a maneuverable target in longtime tracking scenario by a quad rotor, thus achieving moving target control. Additionally, Haibo Du and Chunjiang Qian proposed a finite-time controller to solve finite-time attitude tracking problem for single spacecraft in [24]. Finite-time tracking control for extended nonholonomic chained-form systems with external disturbance and parametric uncertainty has been considered by Chen et al. [25-27]. Nevertheless, moving target control scheme has seldom been utilized combining extended nonholonomic chained-form systems with external disturbances as represented in the published reports.

For the sake of eliminating the moving target control problem concerning a dynamic output tracking error model, which combines moving target and extended nonholonomic chained-form systems, this paper puts forward a more general target tracking implementation using finite-time controllers. After experimental verification, the designed controllers are proved able to stabilize dynamic output tracking error system with the relative position of the target and external disturbance.

The main contributions in this paper can be summarized as the advance of the moving target tracking scheme. In essence, moving target is arbitrary moving particle, while the ideal trajectory of traditional tracking control must have exactly the same kinematics equation as the original robots.

Comparing with traditional tracking, the tracking scheme designed for moving target is capable of being more extensively applied to the more general targets, rather than just tracking immovable targets as usual. Specifically, the novelty of the control technique proposed for practical moving target can be summarized to the following three aspects. Firstly, this paper focuses on the tracking problem of the more general situations to ensure mostly moving targets be successfully tracked, which is different from the traditional tracking problem requiring consistent constraints in the kinematics system. Secondly, the extended chained system is based on dynamics with force and moment design as the controller, comparing with kinematics based on speed as the controller. Thirdly, we adopt finite-time switching control scheme to eliminate the moving target control problem, which has the optimal convergence speed with better robust performance and antidisturbance performance. Meanwhile, discontinuous switching controllers are designed by splitting the chained-form system into two subsystems, then stabilizing the dynamic output tracking error system strictly.

The main work of this article is organized as the following points.

(1) Section 1 develops a dynamic output tracking error model combining moving target and extended dynamic nonholonomic chained-form systems to achieve trajectory tracking without nonholonomic constraints regarding the more general and more practical moving target tracking situations.

(2) Section 2 explains the detailed procedure of controller designing with the stability analysis through splitting the chained-form tracking error system to two decoupled subsystems, then accomplishing switching control according to the finite-time stability control theory.

(3) Finally, several simulations provide the corresponding experimental results concerning the proposed finite-time switching control methodology in Section 3. According to the verification above, the stabilization regarding the tracking error model is ensured successfully.

\section{Problem Statement}

Considering moving target tracking problem, the paper considers extended nonholonomic chained-form systems as the initial model. Nonholonomic systems in the extended chain formal model are given as follows:

$$
\begin{aligned}
& \dot{x}_{1}=u_{1} \\
& \dot{x}_{2}=x_{3} u_{1} \\
& \dot{x}_{3}=u_{2} \\
& \dot{u}_{1}=\tau_{1}+d_{1}(x, t) \\
& \dot{u}_{2}=\tau_{2}+d_{2}(x, t)
\end{aligned}
$$

where $x_{1} \in R^{\mathrm{n}}, x_{2} \in R^{\mathrm{n}}, x_{3} \in R^{\mathrm{n}}$ represent different state vectors in the chained-form system, $u_{1} \in R^{2}, u_{2} \in R^{2}$ are two velocity inputs in the kinematics model and so on $[1,2]$. Besides, the practical control inputs $\tau_{1} \in R^{2}, \tau_{2} \in R^{2}$ represent two formal inputs of force or torque in the extended dynamic model [28-30].

Practically, the target model does not satisfy nonholonomic constraints necessarily; therefore, we design a more practical tracking system combining moving target and extended dynamic nonholonomic chained-form systems in the description, which seems to be more widely applied to general situations. The target model here is described as

$$
\begin{aligned}
& \dot{x}_{1 r}=f_{1}\left(x_{r}, t\right) \\
& \dot{x}_{2 r}=f_{2}\left(x_{r}, t\right)
\end{aligned}
$$

where $\left[x_{1 r}, x_{2 r}\right]^{T} \in R^{2}$ are the position of the target, $f_{1}\left(x_{r}, t\right)$, $f_{2}\left(x_{r}, t\right), \forall\left(x_{r}, t\right) \in R$ are velocities of target. Defining the dynamics of tracking error $e_{1}=x_{1}-x_{1 r} \in R^{n}, e_{2}=$ $x_{2}-x_{2 r} \in R^{n}$ on the basis of manipulations from (1) and (2), then tracking error system can be proved as the following description:

$$
\begin{aligned}
& \dot{e}_{1}=\dot{x}_{1}-\dot{x}_{1 r} \\
& \dot{e}_{2}=\dot{x}_{2}-\dot{x}_{2 r} \\
& \dot{x}_{3}=u_{2} \\
& \dot{u}_{1}=\tau_{1}+d_{1}(x, t) \\
& \dot{u}_{2}=\tau_{2}+d_{2}(x, t)
\end{aligned}
$$

From a practical standpoint, the velocities of the moving target and the external disturbances must be bounded; hence, we make the following reasonable assumptions. 
Assumption 1. The time-varying external uncertain disturbances $d_{i}(x, t) \in R,(i=1,2)$ are bounded and differentiable, satisfying that

$$
\begin{array}{r}
\left|d_{i}(x, t)\right| \leq \bar{d}_{i}, \\
\left|\dot{d}_{i}(\cdot)\right| \leq a_{i c}, \quad a_{i c}>0 \\
(i=1,2)
\end{array}
$$

with known bounds $\bar{d}_{i}, a_{i c} \in R^{+}(i=1,2)$ given in advance.

Assumption 2. The velocities of target $f_{1}\left(x_{r}, t\right), f_{2}\left(x_{r}, t\right)$, $\forall\left(x_{r}, t\right) \in R$ satisfying $f_{1}\left(x_{r}, t\right) \neq 0, f_{2}\left(x_{r}, t\right) \neq 0$.

Moreover, $\dot{f}_{1}\left(x_{r}, t\right), \dot{f}_{2}\left(x_{r}, t\right), \ddot{f}_{1}\left(x_{r}, t\right), \ddot{f}_{2}\left(x_{r}, t\right)$ are supposed to be available.

Next, in order to present our controllers design, the following two lemmas are needed.

Lemma 3 (see [31]). For the following system,

$$
\begin{aligned}
\dot{\bar{x}} & =f(\bar{x}), \\
f(0) & =0,
\end{aligned}
$$

$$
\bar{x} \in R^{n}
$$

suppose there exists a continuous function $\bar{V}(\bar{x}): U \longrightarrow R$, and the following statements hold.

(1) $\bar{V}(\bar{x})$ is positive definitely.

(2) There exist real numbers $\bar{c}>0, \bar{\alpha} \in(0,1)$ and an open neighborhood $U_{0} \in U$ of the origin, such that the inequality $\dot{\bar{V}}(\bar{x})+\bar{c}^{\bar{c}}(\bar{x}) \leq 0, \bar{x} \in U_{0} \backslash\{0\}$ is true.

Then the origin is a stable equilibrium of system (5) in finite time. Besides, if $U=U_{0}=R^{n}$, then the origin is a globally stable equilibrium of system (5).

Lemma 4 (see [16]). Consider the time-varying chained-form system:

$$
\begin{gathered}
\dot{z}_{1}=p_{1} z_{2} f(t) \\
\dot{z}_{2}=p_{2} z_{3} f(t) \\
\vdots \\
\dot{z}_{n-1}=p_{n-1} z_{n} f(t) \\
\dot{z}_{n}=\bar{u}
\end{gathered}
$$

where $z=\left[z_{1}, z_{2}, \ldots, z_{n}\right]^{T} \in R^{n}, n \in R$ represents the state vector and $\bar{u} \in R$ is a control input. $f(t): R^{+} \longmapsto R^{+}$is a continuous, bounded function with its boundary $0<\gamma \leq$ $f(t) \leq \widehat{\gamma}$.

If real numbers $l_{1}, r_{i}, \beta_{i-1}>0(i=1,2, \ldots, n$,$) and odd$ integers $p>0, q>0, k=p / q-1<0$ satisfying

$$
\begin{aligned}
& r_{1}=1 \\
& r_{i}=r_{i-1}+k, \quad i=1, \ldots, n, r_{i}>-k>0
\end{aligned}
$$

$$
\begin{aligned}
\beta_{0} & =r_{2} \\
\left(\beta_{i}+1\right) r_{i+1} & =\left(\beta_{i-1}+1\right) r_{i}>0, \quad i=1, \ldots, n-1
\end{aligned}
$$

then the finite time stabilizer of (6) can be given as $\bar{u}=v_{n}=$

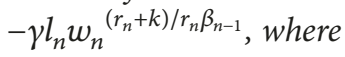

$$
\begin{aligned}
w_{1} & =z_{1}^{1+k} \\
w_{i} & =z_{i}^{\beta_{i-1}}-v_{i-1}{ }^{\beta_{i-1}}, \quad i=2, \ldots, n \\
v_{i-1} & =-l_{i-1} w_{i-1}\left(r_{i-1}+k\right) / r_{i-1} \beta_{i-2} .
\end{aligned}
$$

Based on the preliminary statements, we will give our main results including controller design and stability analysis in the following section.

\section{Main Results}

Controller design and stability analysis are presented in this section. The main idea of controller designing is splitting the chained-form tracking error system to two decoupled subsystems, then accomplishing switching control according to the finite-time stability control theory.

3.1. Controller Design. First of all, on the basis of (1) and (2), (3) can be simplified as

$$
\begin{aligned}
& \dot{e}_{1}=u_{1}-f_{1}\left(x_{r}, t\right) \\
& \dot{e}_{2}=x_{3} u_{1}-f_{2}\left(x_{r}, t\right) \\
& \dot{x}_{3}=u_{2} \\
& \dot{\bar{u}}_{1}=\tau_{1}-\dot{f}_{1}\left(x_{r}, t\right)+d_{1}(x, t) \\
& \dot{u}_{2}=\tau_{2}+d_{2}(x, t)
\end{aligned}
$$

Then, for the convenience of description, we make the following transformation: let $u_{1}-f_{1}\left(x_{r}, t\right)=\bar{u}_{1}$; thus $\dot{\bar{u}}_{1}=$ $\tau_{1}-\dot{f}_{1}\left(x_{r}, t\right)+d_{1}(x, t)$.

Therefore, the final kinematics equations can be represented as

$$
\begin{aligned}
& \dot{e}_{1}=\bar{u}_{1} \\
& \dot{e}_{2}=x_{3} u_{1}-f_{2}\left(x_{r}, t\right) \\
& \dot{x}_{3}=u_{2} \\
& \dot{\bar{u}}_{1}=\tau_{1}-\dot{f}_{1}\left(x_{r}, t\right)+d_{1}(x, t) \\
& \dot{u}_{2}=\tau_{2}+d_{2}(x, t)
\end{aligned}
$$

Next, we depart the system above into two subsystems (11) and (12), respectively:

$$
\begin{aligned}
& \dot{e}_{1}=\bar{u}_{1} \\
& \dot{\bar{u}}_{1}=\tau_{1}-\dot{f}_{1}\left(x_{r}, t\right)+d_{1}(x, t)
\end{aligned}
$$


and

$$
\begin{aligned}
& \dot{e}_{2}=x_{3} u_{1}-f_{2}\left(x_{r}, t\right) \\
& \dot{x}_{3}=u_{2} \\
& \dot{u}_{2}=\tau_{2}+d_{2}(x, t)
\end{aligned}
$$

The design approach in regard to the above problem is described as follows. If a chattering-free control $\tau_{1}$ is designed for the above system (10), then there exists a finite-time $T_{1}<$ $+\infty$ satisfying $\bar{u}_{1}=0, e_{1}=0$ as $t \geq T_{1}$, thus achieving $\left(e_{1}, \bar{u}_{1}\right) \longrightarrow 0$.

After $\tau_{1}$ is proved available, the first subsystem is bound to stabilize to zero. Therefore, it is obvious that $\bar{u}_{1} \longrightarrow 0$; thus $u_{1}=f_{1}\left(x_{r}, t\right)$. Then, the second subsystem (12) can be simplified as

$$
\begin{aligned}
& \dot{e}_{2}=x_{3} f_{1}\left(x_{r}, t\right)-f_{2}\left(x_{r}, t\right) \\
& \dot{x}_{3}=u_{2} \\
& \dot{u}_{2}=\tau_{2}+d_{2}(x, t)
\end{aligned}
$$

Then, we make such transformation letting $\bar{x}_{3}=x_{3} f_{1}\left(x_{r}, t\right)-$ $f_{2}\left(x_{r}, t\right)$; thus $\dot{\bar{x}}_{3}=u_{2} f_{1}\left(x_{r}, t\right)+x_{3} \dot{f}_{1}\left(x_{r}, t\right)-\dot{f}_{2}\left(x_{r}, t\right)$. Then, we introduce $\bar{u}_{2}$ to replace $u_{2} f_{1}\left(x_{r}, t\right)+x_{3} \dot{f}_{1}\left(x_{r}, t\right)-\dot{f}_{2}\left(x_{r}, t\right)$; the equation can be transformed to

$$
\begin{aligned}
\dot{e}_{2}= & \bar{x}_{3} \\
\dot{\bar{x}}_{3}= & \bar{u}_{2} \\
\dot{\bar{u}}_{2}= & {\left[\tau_{2}+d_{2}(x, t)\right] f_{1}\left(x_{r}, t\right)+2 u_{2} \dot{f}_{1}\left(x_{r}, t\right) } \\
& +x_{3} \ddot{f}_{1}\left(x_{r}, t\right)-\ddot{f}_{2}\left(x_{r}, t\right)
\end{aligned}
$$

Next, the following part is concentrated on main conclusion.

3.2. Stability Analysis. In order to give reasonable stability analysis, we introduce Lyapunov function and utilize its time derivative to prove it effectively and strictly.

Theorem 5. Given positive constants $\delta_{i}, b_{i}, a_{i q}>0,(i=1,2)$, satisfying $a_{i q} \geq b_{i} \bar{c}_{i}, \bar{a}_{2 c} \geq a_{2 c}+\bar{c}_{2}$.

For tracking error system (3), take the following two discontinuous switching controllers:

$$
\begin{aligned}
\tau_{1}= & \tau_{1 h}+\tau_{1 r}+\dot{f}_{1}\left(x_{r}, t\right) \\
\tau_{2} & \\
= & \frac{\tau_{2 h}+\tau_{2 r}-2 u_{2} \dot{f}_{1}\left(x_{r}, t\right)-x_{3} \ddot{f}_{1}\left(x_{r}, t\right)+\ddot{f}_{2}\left(x_{r}, t\right)}{f_{1}\left(x_{r}, t\right)}
\end{aligned}
$$

where

$$
\begin{aligned}
& \tau_{1 h}=\bar{v}_{2}, \\
& \bar{v}_{2}=-l_{2} \bar{w}_{2}{ }^{\left(r_{2}+a\right) / r_{2} \beta_{1}}, \\
& \bar{w}_{2}=\bar{u}_{1}{ }^{\beta_{1}}-\bar{v}_{1} \beta_{1} \\
& \bar{w}_{1}=e_{1}{ }^{1+a}, \\
& \bar{v}_{1}=-l_{1} \bar{w}_{1}{ }^{\left(r_{1}+a\right) / r_{1} \beta_{0}}
\end{aligned}
$$

$$
\begin{aligned}
\dot{\tau}_{1 r}+b_{1} \tau_{1 r} & =-\left(a_{1 c}+a_{1 b}+\delta_{1}\right) \operatorname{sgn}\left(\varepsilon_{1}\right) \\
\varepsilon_{1} & =\dot{\bar{u}}_{1}-\bar{v}_{2}, \\
\tau_{1 r}(0) & =0
\end{aligned}
$$

and

$$
\begin{aligned}
& \tau_{2 h}=\bar{v}_{3}, \\
& \bar{v}_{3}=-l_{3} \bar{w}_{3}{ }^{\left(r_{3}+a\right) / r_{3} \beta_{2}}, \\
& \bar{w}_{3}=\bar{u}_{2}^{\beta_{2}}-\bar{v}_{2}^{\beta_{2}} \\
& \bar{v}_{2}=-l_{2} \bar{w}_{2}^{\left(r_{2}+a\right) / r_{2} \beta_{1}}, \\
& \bar{w}_{2}=\bar{u}_{2}^{\beta_{1}}-\bar{v}_{1}^{\beta_{1}} \\
& \bar{v}_{1}=-l_{1} \bar{w}_{1}^{\left(r_{1}+a\right) / r_{1} \beta_{0}}, \\
& \bar{w}_{1}=e_{2}{ }^{1+a} \\
& \dot{\tau}_{2 r}+b_{2} \tau_{2 r}=-\left(\bar{a}_{2 c}+a_{2 b}+\delta_{2}\right) \operatorname{sgn}\left(\varepsilon_{2}\right) \\
& \varepsilon_{2}=\dot{\bar{u}}_{2}-\bar{v}_{3}, \\
& \tau_{2 r}(0)=0
\end{aligned}
$$

Then tracking error system (3) can be stabilized to zero within a finite time.

Proof. For system (15), we introduce $\bar{\tau}_{1}$ to replace $\tau_{1}$ $\dot{f}_{1}\left(x_{r}, t\right), \bar{\tau}_{2}$ to replace $\tau_{2} f_{1}\left(x_{r}, t\right)+2 u_{2} \dot{f}_{1}\left(x_{r}, t\right)+x_{3} \ddot{f}_{1}\left(x_{r}, t\right)-$ $\ddot{f}_{2}\left(x_{r}, t\right)$; then the state feedback control law can be transformed into

$$
\begin{aligned}
& \bar{\tau}_{1}=\tau_{1 h}+\tau_{1 r} \\
& \bar{\tau}_{2}=\tau_{2 h}+\tau_{2 r}
\end{aligned}
$$

As for system (11), substituting $\tau_{1}$ from (16) and (18), we obtain

$$
\begin{aligned}
\dot{e}_{1} & =\bar{u}_{1} \\
\dot{\bar{u}}_{1} & =\tau_{1 h}+\tau_{1 r}+d_{1}(x, t)
\end{aligned}
$$

Next, substituting $\tau_{1 h}$ from (16) to (19),

$$
\begin{aligned}
& \dot{e}_{1}=\bar{u}_{1} \\
& \dot{\bar{u}}_{1}=\bar{v}_{2}+\tau_{1 r}+d_{1}(x, t)
\end{aligned}
$$

By Lemma $4, \bar{v}_{2}$ represents a finite-time stability controller supporting sliding-mode surface dynamics [25]:

$$
\begin{aligned}
& \dot{e}_{1}=\bar{u}_{1} \\
& \dot{\bar{u}}_{1}=\bar{v}_{2}
\end{aligned}
$$

Based on (19), denote a sliding-mode variable equation is obvious as follows:

$$
\varepsilon_{1}:=\dot{\bar{u}}_{1}-\bar{v}_{2}=\tau_{1 r}+d_{1}(x, t)
$$


In case of $\varepsilon_{1}=0$, nonlinear system (20) will stabilize to zero within a finite time.

Here, we introduce Lyapunov function as follows about system (20) for further certification:

$$
V_{1}=\frac{1}{2} \varepsilon_{1}^{2}
$$

Its time derivative is given as follows:

$$
\begin{aligned}
\dot{V}_{1} & =\varepsilon_{1} \dot{\varepsilon}_{1}=\varepsilon_{1}\left(\dot{\tau}_{1 r}+\dot{d}_{1}(x, t)\right) \\
& =\varepsilon_{1}\left[-b_{1} \tau_{1 r}-\left(a_{1 c}+a_{1 b}+\delta_{1}\right) \operatorname{sgn}\left(\varepsilon_{1}\right)+\dot{d}_{1}(x, t)\right] \\
& =-\left(a_{1 c}+a_{1 b}+\delta_{1}\right)\left|\varepsilon_{1}\right|+\dot{d}_{1}(x, t) \varepsilon_{1}-b_{1} \tau_{1 r} \varepsilon_{1} \\
& \leq-\left(a_{1 c}+a_{1 b}+\delta_{1}\right)\left|\varepsilon_{1}\right|+a_{1 c}\left|\varepsilon_{1}\right|-b_{1} \tau_{1 r} \varepsilon_{1}
\end{aligned}
$$

Through simplification, the following equation can obtain the following:

$$
\dot{V}_{1} \leq-\left(a_{1 b}+\delta_{1}\right)\left|\varepsilon_{1}\right|-b_{1} \tau_{1 r} \varepsilon_{1}
$$

Meanwhile, combining (16), $\tau_{1 r}$ can be calculated as

$$
b_{1} \tau_{1 r}=-\left(a_{1 c} a_{1 b}+\delta_{1}\right) \operatorname{sgn}\left(\varepsilon_{1}\right)\left(1-e^{-b_{1} t}\right)
$$

Considering the initial condition $\tau_{1 r}(0)=0$, combining (22) and (26), the following posture can be proved:

$$
a_{1 b} \geq b_{1} \bar{c}_{1} \geq b_{1} \max \left(\tau_{1 r}\right) \geq b_{1}\left|\tau_{1 r}\right|
$$

Considering the above certification, we finally get

$$
\begin{aligned}
\dot{V}_{1} & =\varepsilon_{1} \dot{\varepsilon}_{1} \leq\left(a_{1 b}+\delta_{1}\right)\left|\varepsilon_{1}\right|+b_{1}\left|\tau_{1 r}\right|\left|\varepsilon_{1}\right| \leq-\delta_{1}\left|\varepsilon_{1}\right| \\
& =-\sqrt{2} \delta_{1}\left(V_{1}\right)^{1 / 2} \leq 0
\end{aligned}
$$

Hence, subsystem (19) is proved to be capable of stabilizing to the desirable sliding-mode dynamic surface (21), when there exists a finite time $T_{1}<+\infty$ satisfying $e_{1}=0, \bar{u}_{1}=0$ as $t \geq T_{1}$.

Then turn to the second subsystem (14). To simplify the calculation, $\bar{d}_{2}(x, t)$ is introduced to replace $d_{2}(x, t) f_{1}\left(x_{r}, t\right)$, and the constant $\bar{d}_{2}(x, t)$ is restrictive on the basis of the mentioned assumptions above. Then we obtain

$$
\begin{aligned}
\dot{e}_{2} & =\bar{x}_{3} \\
\dot{\bar{x}}_{3} & =\bar{u}_{2} \\
\dot{\bar{u}}_{2} & =\bar{\tau}_{2}+\bar{d}_{2}(x, t)
\end{aligned}
$$

Similar to the first system, a desirable stable sliding-mode dynamic surface within finite-time is selected by Lemma 4 .

$$
\begin{aligned}
\dot{e}_{2} & =\bar{x}_{3} \\
\dot{\bar{x}}_{3} & =\bar{u}_{2} \\
\dot{\bar{u}}_{2} & =\bar{v}_{3}
\end{aligned}
$$

Then denote the sliding-mode variable equation as

$$
\varepsilon_{2}:=\dot{\bar{u}}_{2}-\bar{v}_{3}=\tau_{2 r}+d_{2}(x, t)
$$

Similarly, Lyapunov function $V_{2}=(1 / 2) \varepsilon_{2}{ }^{2}$ is introduced about system (20) for further certification. Through simple calculation, its derivative regarding time $t$ along (29) is as follows:

$$
\begin{aligned}
\dot{V}_{2} & =\varepsilon_{2} \dot{\varepsilon}_{2}=\varepsilon_{2}\left(\dot{\tau}_{2 r}+\dot{d}_{2}(x, t)\right) \\
& \left.=\varepsilon_{2} \mid-b_{2} \tau_{2 r}-\left(\bar{a}_{2 c}+a_{2 b}+\delta_{2}\right) \operatorname{sgn}\left(\varepsilon_{2}\right)+\dot{d}_{2}(x, t)\right\rfloor \\
& \leq-\left(\bar{a}_{2 c}+a_{2 b}+\delta_{2}\right)\left|\varepsilon_{2}\right|+a_{2 c}\left|\varepsilon_{2}\right|+b_{2}\left|\tau_{2 r}\right|\left|\varepsilon_{2}\right| \\
& =-\delta_{2}\left|\varepsilon_{2}\right|-\left(\bar{a}_{2 c}-a_{2 c}\right)\left|\varepsilon_{2}\right|-\left(a_{2 b}-b_{2}\left|\tau_{2 r}\right|\right)\left|\varepsilon_{2}\right|
\end{aligned}
$$

Combining (15) and (31), considering $a_{2 b} \geq b_{2} \bar{c}_{2} \geq$ $b_{2} \max \left(\tau_{2 r}\right) \geq b_{2}\left|\tau_{2 r}\right|$, the following inequality can be launched:

$$
\begin{aligned}
\dot{V}_{2} & =\varepsilon_{2} \dot{\varepsilon}_{2} \leq \delta_{2}\left|\varepsilon_{2}\right|-\left(a_{2 b}-b_{2}\left|\tau_{2 r}\right|\right)\left|\varepsilon_{2}\right| \leq-\delta_{2}\left|\varepsilon_{2}\right| \\
& =-\sqrt{2} \delta_{2}\left(V_{2}\right)^{1 / 2} \leq 0
\end{aligned}
$$

Therefore, the mentioned subsystem (20) can be stable to the desirable sliding-mode dynamic surface (30) within a finite time $T_{2}<+\infty$ satisfying $e_{2}=e_{3}=0$ as $t \geq T_{2}+T_{1}$.

In summary, the initial tracking error system denoted in (3) is capable of stabilizing to zero in a finite time $T=T_{1}+T_{2}<$ $+\infty$ as $t \longrightarrow T$, along with $e_{i} \equiv 0,(i=1, \ldots, n), \forall t \geq T$.

Remark 6. Note that the sign functions are designed on the right side of $\dot{\tau}_{1 r}, \dot{\tau}_{2 r}$. Therefore, controller (18) is nonsmooth but continuous in anti-interference components $\tau_{1 r}, \tau_{2 r}$ [26]. Additionally, we consider $\tau_{1 r}, \tau_{2 r}$ as the outputs of filters $\dot{\tau}_{1 r}=-b_{i} \tau_{i r}-v_{i}(t), v_{i}(t)=-\left(a_{i c}+a_{i b}+\delta_{i}\right) \operatorname{sgn}\left(\varepsilon_{i}(t)\right), i=$ 1,2. Meanwhile, $\tau_{1 r}, \tau_{2 r}$ are softened into continuous signals. According to the following Laplace transfer functions, which are applicable to the filters,

$$
\frac{\tau_{i r}(s)}{v_{i}(s)}=\frac{1}{s+b_{i}}, \quad i=1,2
$$

Therefore, chattering in traditional sliding-mode design can be averted with continuous controllers $\tau_{1 r}, \tau_{2 r}$.

Remark 7. The sliding-mode variables $\varepsilon_{i}(i=1,2)$ are not exactly available. Nevertheless, it is not difficult to obtain $\operatorname{sgn}\left(\varepsilon_{i}\right)$ for implementing the controllers with condition $\varepsilon_{i}>0$ or $\varepsilon_{i}<0$. For instance, if there exists a retrievable function about $\varepsilon_{i}$,

$$
\begin{aligned}
f(t) & =\int_{0}^{t} \varepsilon_{1}(s) d s=\int_{0}^{t}\left(\dot{\bar{u}}_{1}-\bar{v}_{2}\right) d s \\
& =\bar{u}_{1}(t)-\bar{u}_{0}(t)+\int_{0}^{t} l_{2} \bar{w}_{2}{ }^{\left(r_{2}+\alpha\right) / r_{2} \beta_{1}}(s) d s,
\end{aligned}
$$

the following equation can be proved

$$
\varepsilon_{1}(t)=\lim _{\vartheta \longrightarrow 0} \frac{h(t+\vartheta)-h(t)}{\vartheta}
$$




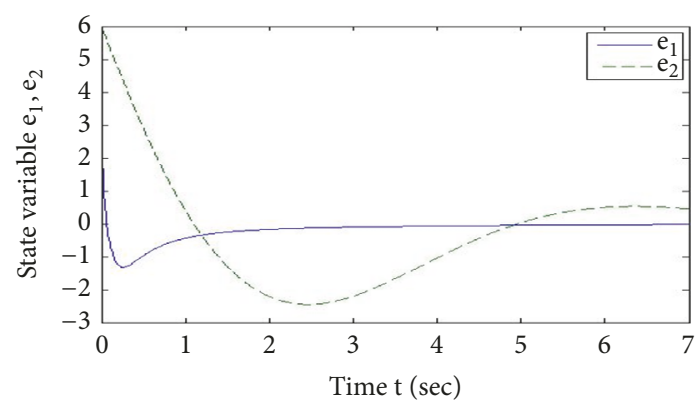

FIGURE 1: Target tracking process for the first step.

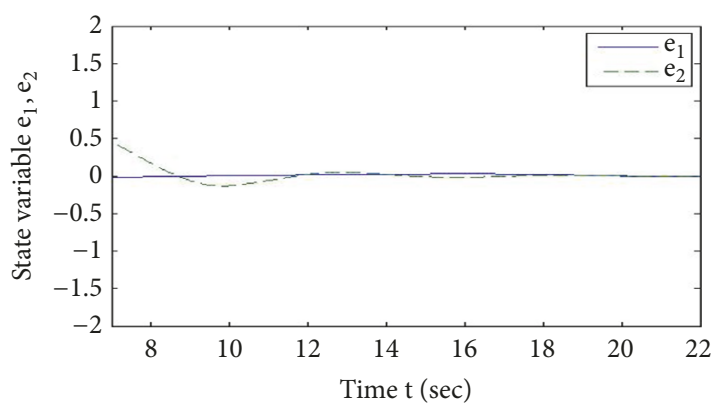

FIGURE 2: Target tracking process for the second step.

where $\vartheta$ is a tiny time sampling period; meanwhile $\operatorname{sgn}\left(\varepsilon_{1}\right)$ can be obtained by $h(t+\vartheta)-h(t)$. By the above calculation, $\operatorname{sgn}\left(\varepsilon_{2}\right)$ can be obtained in the same way.

\section{Simulations}

In this section, the proposed controller is adopted to track the motion trajectory of the target. Here we prove the effectiveness of the above method by MATLAB simulation.

In the following simulation, the system is divided into two subsystems (20) and (29). For the first subsystem, we assume $\alpha=1, \beta_{0}=5 / 7, \beta_{1}=7 / 5, r_{1}=1, r_{2}=2, l_{1}=l_{2}=1$. Then, we choose parameters of $\tau_{1 r}: b_{1}=2.2, a_{1 b}=0.3, a_{1 c}=0.5$, $\delta_{1}=1$. For the second subsystem, we assume $\alpha=1, \beta_{0}=5 / 7$, $\beta_{1}=7 / 5, \beta_{2}=1, r_{1}=1, r_{2}=5 / 7, r_{3}=3 / 7, l_{1}=l_{2}=l_{3}=1$. We choose the parameters of $\tau_{2 r}: b_{2}=1, a_{2 b}=0.7, \bar{a}_{2 c}=1.3$, $\delta_{2}=1$.

On the basis of the above parameters, we simulate the target tracking process of two subsystems.

By the numeric simulations regarding Figures 1-3, we conclude that all of these subsystems can stabilize to zero.

Figure 1 shows the first subsystem can converge to zero in a finite time $t<7 s$. At this time, the second subsystems are still fluctuating. After 7 seconds, the first subsystem is shown to stabilize to zero.

Figure 2 shows that the first subsystem has completely converge to zero in a finite time $7 s<t<22 s$. At this time, the second subsystems begin to stabilize and the amplitude begins to decaying. After 22 seconds, the two subsystems stabilize to zero, and then the target tracking is achieved.

Figure 3 shows the total process of two subsystems within 0 to 40 seconds, respectively. We can conclude that

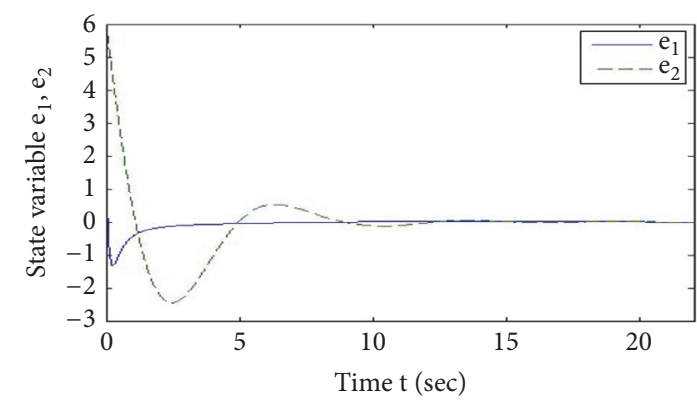

FIgURE 3: General process.

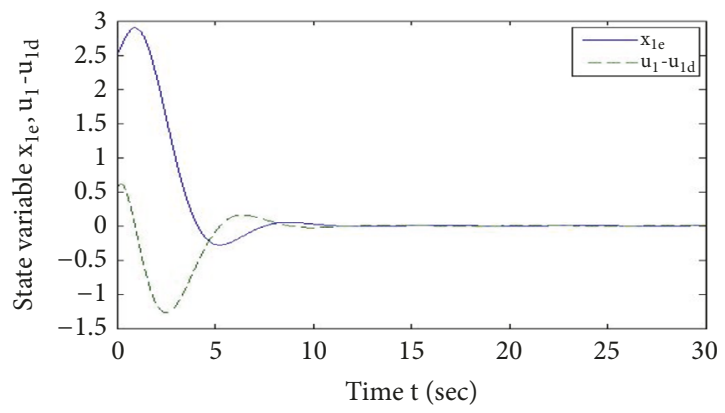

FIGURE 4: The response of state variable $\left(x_{1 e}, u_{1}-u_{1 d}\right)$ with respect to time.

the first subsystem will stable to 0 faster, and then the second subsystems begin to approach and later stabilize at 0 .

According to the comparison with some recent papers like Chen [23], we give numeric simulations with the same initial condition $(2.5,6,2,-2)$ for their sliding-mode controller in Figures 4 and 5. We conclude that our controller can perform better than theirs. Since our two subsystems can stabilize at 0 to 7 seconds and 0 to 22 seconds, separately. Additionally, their tracking error state will converge to zero in $t<12 s$ and $t<25 s$.

\section{Conclusion}

The finite-time tracking a practical moving target problem is considered for the extended nonholonomic chained-form systems. For the dynamic output tracking error model, two decoupled subsystems are proposed, based on which the rigorous convergence and stability analysis are presented by applying the finite-time stability control theory and switching design methods. And finally, the effectiveness of the proposed finite-time switching control approach is verified by the simulation results.

In our future research, we will make further exploration, concentrating on transitioning from theoretical research to the realization of practical applications step by step, achieving experimental for the practical system. 


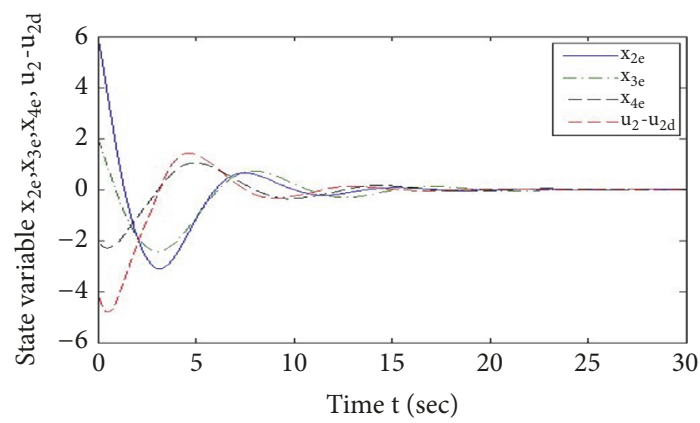

Figure 5: The response of state variable $\left(x_{2 e}, x_{3 e}, x_{4 e}, u_{2}-u_{2 d}\right)$ with respect to time.

\section{Data Availability}

The source code of simulation research used to support the findings of this study are available from the corresponding author upon request.

\section{Conflicts of Interest}

The authors declare that they have no conflicts of interest.

\section{Acknowledgments}

This paper was supported by the Natural Science Foundation of China (61304004 and 61503205), the Changzhou Sci\&Tech Program (CJ20160013), the Fundamental Research Funds for the Central Universities (2017B15114), and the Changzhou Key Laboratory of Aerial Work Equipment and Intellectual Technology (CLAI201803).

\section{References}

[1] S. Mobayen, "Finite-time tracking control of chained-form nonholonomic systems with external disturbances based on recursive terminal sliding mode method," Nonlinear Dynamics, vol. 80, no. 1-2, pp. 669-683, 2015.

[2] S. Mobayen and S. Javadi, "Disturbance observer and finite-time tracker design of disturbed third-order nonholonomic systems using terminal sliding mode," Journal of Vibration and Control, vol. 23, no. 2, pp. 181-189, 2015.

[3] M. Janiak and K. Tchoń, "Constrained motion planning of nonholonomic systems," Systems \& Control Letters, vol. 60, no. 8, pp. 625-631, 2011.

[4] P. Svestka and J. Vleugels, "Exact motion planning for tractortrailer robots," in Proceedings of the 1995 IEEE International Conference on Robotics and Automation. Part 1 (of 3), pp. 24452450, Nagoya, Japan, May 1995.

[5] Z. Miao, Y. Wang, and R. Fierro, "Cooperative circumnavigation of a moving target with multiple nonholonomic robots using backstepping design," Systems \& Control Letters, vol. 103, pp. 5865, 2017.

[6] M. Ou, H. Du, and S. Li, "Finite-time tracking control of multiple nonholonomic mobile robots," Journal of The Franklin Institute, vol. 349, no. 9, pp. 2834-2860, 2012.
[7] I. Kolmanovsky and N. H. McClamroch, "Developments in nonholonomic control problems," IEEE Control Systems Magazine, vol. 15, no. 6, pp. 20-36, 1995.

[8] J. Ghommam, H. Mehrjerdi, F. Mnif, and M. Saad, "Cascade design for formation control of nonholonomic systems in chained form," Journal of The Franklin Institute, vol. 348, no. 6, pp. 973-998, 2011.

[9] Z.-Y. Liang and C.-L. Wang, "Robust stabilization of nonholonomic chained form systems with uncertainties," Acta Automatica Sinica, vol. 37, no. 2, pp. 129-142, 2011.

[10] D. Y. Chen, Y. X. Liu, X. Y. Ma, and R. F. Zhang, "Control of a class of fractional-order chaotic systems via sliding mode," Nonlinear Dynamics, vol. 67, no. 1, pp. 893-901, 2012.

[11] S. Mobayen, "Finite-time robust-tracking and model-following controller for uncertain dynamical systems," Journal of Vibration and Control, vol. 22, no. 4, pp. 169-182, 2016.

[12] M. Sun, Y. Qian, and J. Chen, "Repetitive learning finite-time control," in Proceedings of the 27th Chinese Control and Decision Conference, CCDC 2015, pp. 1772-1777, May 2015.

[13] J. Wang, T. Gao, and G. Zhang, "Adaptive finite-time control for hyperchaotic Lorenz-Stenflo systems," Physica Scripta, vol. 90, no. 2, 2015.

[14] L. Shihua, D. Shihong, and Q. Li, "Global set stabilisation of the spacecraft attitude using finite-time control technique," International Journal of Control, vol. 82, no. 5, pp. 822-836, 2009.

[15] C. Qian and J. Li, "Global finite-time stabilization by output feedback for planar systems without observable linearization," IEEE Transactions on Automatic Control, vol. 50, no. 6, pp. 885890, 2005.

[16] Y. Hong, J. Wang, and Z. Xi, "Stabilization of uncertain chained form systems within finite settling time," IEEE Transactions on Automatic Control, vol. 50, no. 9, pp. 1379-1384, 2005.

[17] S. Mobayen, "An adaptive fast terminal sliding mode control combined with global sliding mode scheme for tracking control of uncertain nonlinear third-order systems," Nonlinear Dynamics, vol. 82, no. 1, pp. 1-12, 2015.

[18] S.-O. Lee, Y.-J. Cho, M. Hwang-Bo, B.-J. You, and S.-R. Oh, "A stable target-tracking control for unicycle mobile robots," in Proceedings of the 2000 IEEE/RSJ International Conference on Intelligent Robots and Systems (IROS 2000), vol. 3, pp. 1822-1827, 2000.

[19] R. Zheng, Y. Liu, and D. Sun, "Enclosing a target by nonholonomic mobile robots with bearing-only measurements," Automatica, vol. 53, pp. 400-407, 2015.

[20] L. Huang, "Control approach for tracking a moving target by a wheeled mobile robot with limited velocities," IET Control Theory \& Applications, vol. 3, no. 12, pp. 1565-1577, 2009.

[21] V. Gazi and R. Ordonez, "Target tracking using artificial potentials and sliding mode control," International Journal of Control, vol. 80, no. 10, pp. 1626-1635, 2007.

[22] Y. Wang, D. Wang, and B. C. Ng, "Finite time moving target tracking using nonholonomic vehicles with distance and bearing angle constraints," in Proceedings of the 2017 American Control Conference, ACC 2017, pp. 2962-2967, Seattle, WA, USA, May 2017.

[23] M. S. Movafegh, S. M. M. Dehghan, and R. Zardashti, "A ThreeDimensional Guidance and Control for Ground Moving Target Tracking," Transactions of the Institute of Measurement and Control, 2018.

[24] H. Du, S. Li, and C. Qian, "Finite-time attitude tracking control of spacecraft with application to attitude synchronization," IEEE 
Transactions on Automatic Control, vol. 56, no. 11, pp. 2711-2717, 2011.

[25] M. Ou, S. Li, and C. Wang, "Finite-time tracking control for multiple non-holonomic mobile robots based on visual servoing," International Journal of Advanced Robotic Systems, vol. 11, no. 10, p. 180, 2014.

[26] H. Chen, B. Zhang, T. Zhao, T. Wang, and K. Li, "Finite-time tracking control for extended nonholonomic chained-form systems with parametric uncertainty and external disturbance," Journal of Vibration and Control, 2016.

[27] C. Canudas de Wit and H. Khennouf, "Quasi-continuous stabilizing controllers for nonholonomic systems: design and robustness consideration," in Proceedings of the 3rd ECC, pp. 2630-2635, 1995.

[28] Y. Wu, B. Wang, and G. D. Zong, "Finite-Time Tracking Controller Design for Nonholonomic Systems with Extended Chained Form," IEEE Transactions on Circuits and Systems II: Express Briefs, vol. 52, no. 11, pp. 798-802, 2005.

[29] H. Chen, "Robust stabilization for a class of dynamic feedback uncertain nonholonomic mobile robots with input saturation," International Journal of Control, Automation, and Systems, vol. 12, no. 6, pp. 1216-1224, 2014.

[30] H. Chen, C. Wang, B. Zhang, and D. Zhang, "Saturated tracking control for nonholonomic mobile robots with dynamic feedback," Transactions of the Institute of Measurement and Control, vol. 35, no. 2, pp. 105-116, 2013.

[31] S. P. Bhat and D. S. Bernstein, "Continuous finite-time stabilization of the translational and rotational double integrators," IEEE Transactions on Automatic Control, vol. 43, no. 5, pp. 678-682, 1998. 


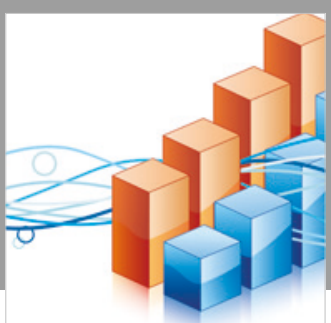

Advances in

Operations Research

\section{-n-m}
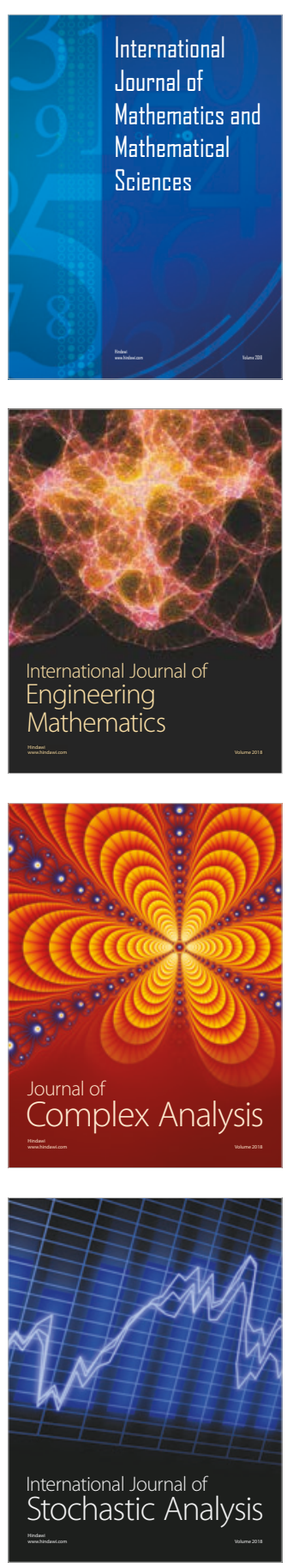
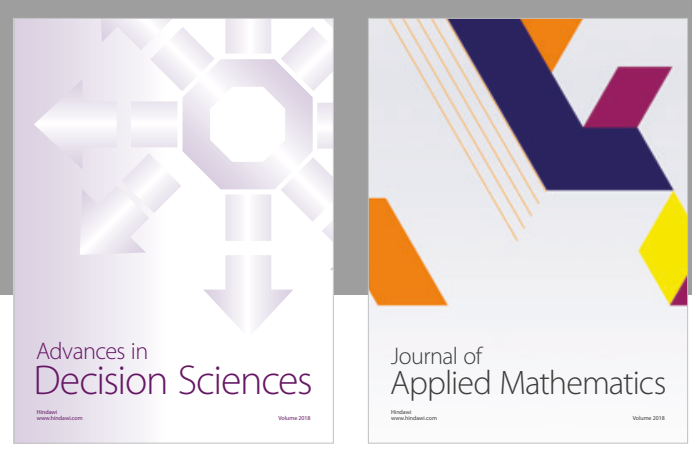

Journal of

Applied Mathematics
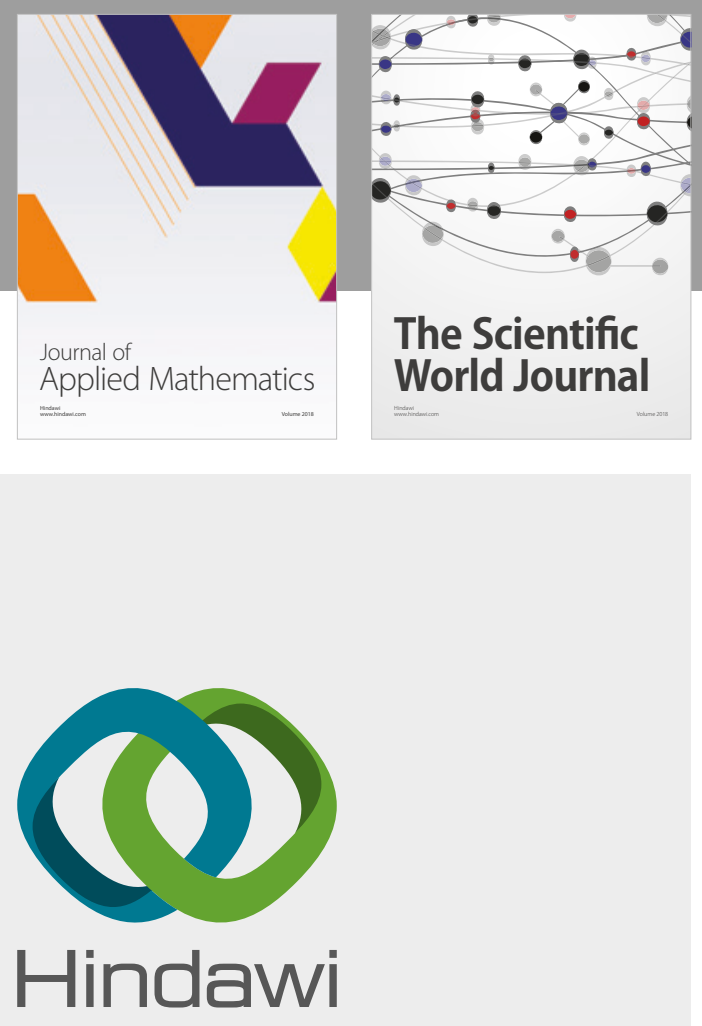

Submit your manuscripts at

www.hindawi.com

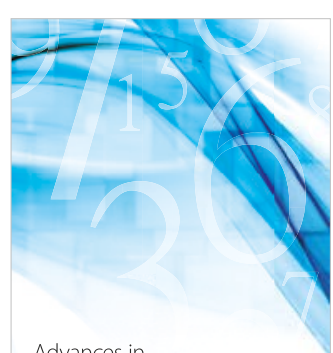

Advances in
Numerical Analysis
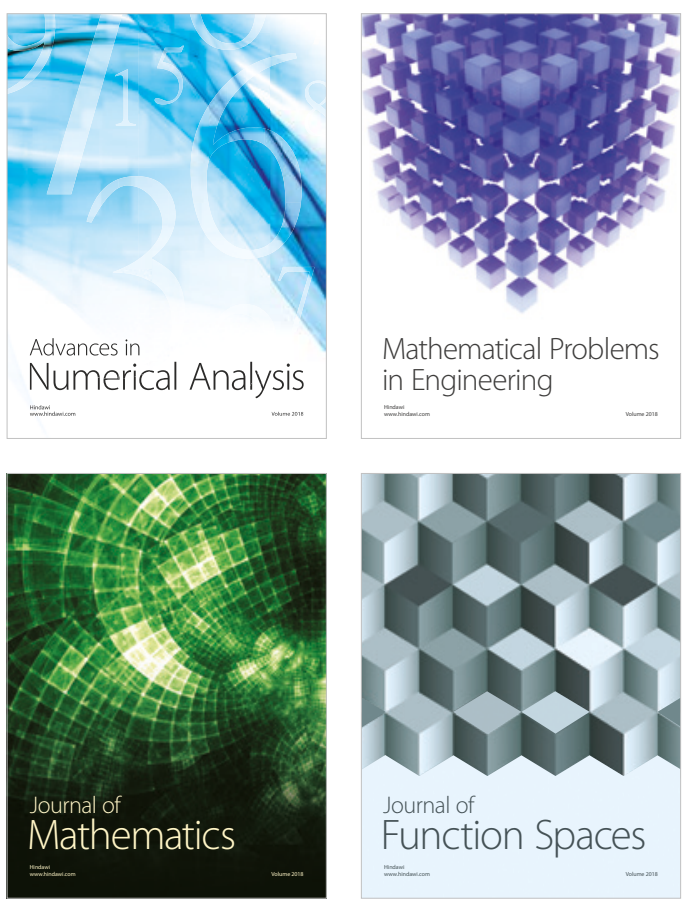

Mathematical Problems in Engineering

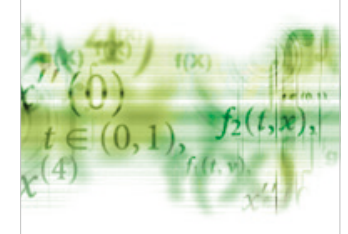

International Journal of

Differential Equations

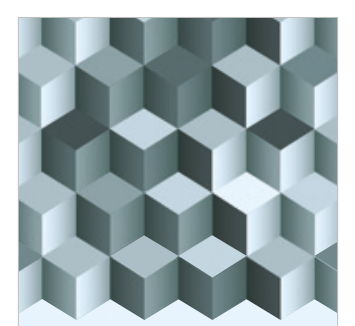

Journal of

Function Spaces

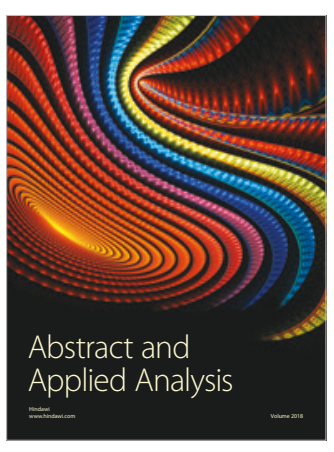

The Scientific

World Journal

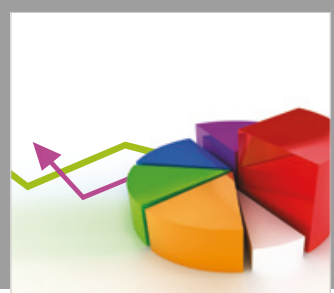

Journal of

Probability and Statistics
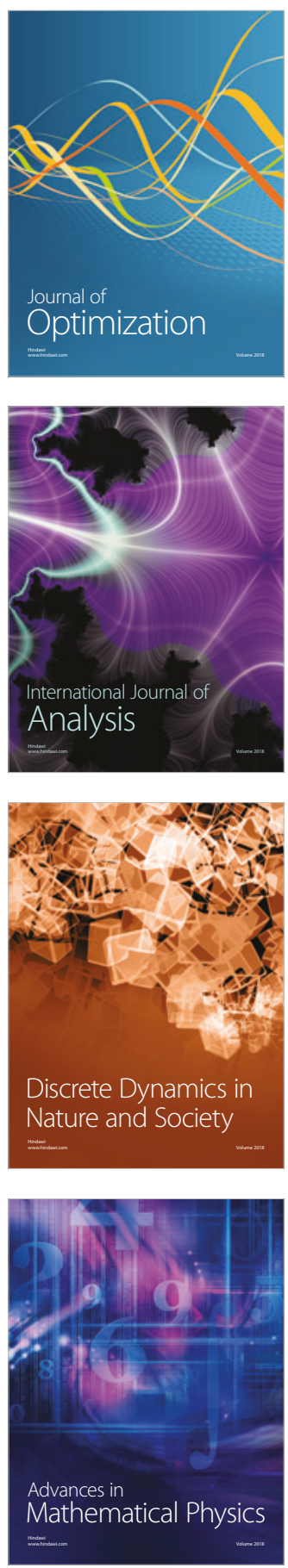